\title{
Surgical Management of Thoracolumbar Myelopathies in Pug Dogs with Concurrent Articular Facet Dysplasia
}

\author{
Anna Tauro ${ }^{1,4}$ Jeremy Rose Rlare Rusbridge $^{3,4}$ \\ ${ }^{1}$ ChesterGates Veterinary Specialists, Neurology Service, Chester, \\ United Kingdom of Great Britain and Northern Ireland \\ 2 Lumbry Park Veterinary Specialists, Neurology Service, Alton, United \\ Kingdom of Great Britain and Northern Ireland \\ ${ }^{3}$ School of Veterinary Medicine, Guildford, United Kingdom of Great \\ Britain and Northern Ireland \\ ${ }^{4}$ Fitzpatrick Referrals, Neurology Service, Eashing, United Kingdom of \\ Great Britain and Northern Ireland
}

Colin J. Driver ${ }^{2,4}$

Address for correspondence Anna Tauro, DVM GpCert(Neuro), MRCVS, ChesterGates Veterinary Specialists, Neurology Service, E \& F, Telford Court, Chestergates Road, Chester CH1 6LT, United Kingdom of Great Britain and Northern Ireland (e-mail: anna.tauro@yahoo.co.uk).

VCOT Open 2019;2:e60-e72.

\begin{abstract}
Keywords

- thoracolumbar myelopathy

- vertebral instability

- arachnoid diverticulum

- pia-arachnoid fibrosis

- intervertebral disc protrusion
\end{abstract}

Objectives The aim of this article is to present the outcome of vertebral stabilization using pins and polymethylmethacrylate in isolation or with concurrent spinal cord decompression for thoracolumbar myelopathies in Pug dogs with associated caudal articular process dysplasia.

Materials and Methods Retrospective case review of 14 Pug dogs with thoracolumbar myelopathy and concurrent caudal articular process dysplasia identified with magnetic resonance imaging and computed tomography. Pug dogs were graded based on the Texas Spinal Cord Injury Scale and clinical follow-up was performed immediately after surgery, at the discharge and at during the postoperative period (median 5 months). Postoperative computed tomography was performed immediately post-surgical intervention in all cases and a variable postoperative time (6 weeks or 6 months). Follow-up with telephone interview with owners was performed at a median time of 12 months postoperatively.

Results Median age of presentation was 8.5 years. In 10/14 cases, neurological improvement was observed, while urinary/faecal incontinence resolved in only two of seven affected cases. Other congenital vertebral malformations were identified in 9/14 cases. Clinical Significance In our population of Pug dogs with thoracolumbar myelopathy and concurrent caudal articular process dysplasia, vertebral stabilization in isolation or with concurrent spinal cord decompression appeared to result in a favourable clinical outcome in the majority of patients. This technique may be favourable for myelopathies with a reportedly poor surgical outcome in this breed.

\section{Introduction}

Chronic progressive thoracolumbar myelopathies have been described in Pug dogs secondary to a variety of conditions including but not exclusive to constrictive myelopathy, piaarachnoid fibrosis, spinal arachnoid diverticulum, intervertebral disc protrusion and dorsal hemivertebrae leading to kyphosis. $^{1-9}$

received

June 29, 2018

accepted after revision

April 16, 2019
DOI https://doi.org/

$10.1055 / \mathrm{s}-0039-1692147$.

ISSN 2625-2325.
Pug dogs appear predisposed to congenital vertebral malformations, including caudal articular process dysplasia, which have been identified in dogs with or without neurological dysfunction (De Decker S. Prevalence and Clinical Relevance of Thoracic Congenital Vertebral Malformations in Screw-Tailed Brachycephalic Dogs. Neurology, Scientific Session in ACVIM, Seattle, Washington, United States, 2018). ${ }^{10-12}$ Caudal articular process dysplasia encompasses congenital anomalies which

\footnotetext{
(c) 2019 Georg Thieme Verlag KG Stuttgart · New York
}

License terms

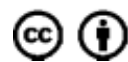


include hypoplastic (partial absence) and aplastic (complete absence) caudal articular processes. It is unclear why caudal articular process dysplasia occurs. ${ }^{13,14}$ While caudal articular process dysplasia can occur at any level of the vertebral column, it is most commonly diagnosed in the T10 to T13 region. ${ }^{10,12}$ Caudal articular process dysplasia has been proposed to result in loss of stability of the vertebral column and has been associated with arachnoid fibrosis, adhesions, tethering of the spinal cord and disruption of normal cerebrospinal fluid circulation. ${ }^{13}$ This has previously been termed 'constrictive myelopathy' (Smiler KL, Patterson JS. Constrictive Myelopathy: a cause of hind limb ataxia unique to Pug dogs? Poster in Tufts' Canine and Feline Breeding and Genetics Conference, Boston, Massachusetts, United States, 2013). ${ }^{8}$ However, biomechanical studies examining the vertebral column of Pug dogs are lacking and an association between caudal articular process dysplasia and the development of constrictive myelopathy has not been proven and remains controversial. Prior trauma has been implicated in the development of constrictive myelopathy in at-risk individuals. ${ }^{8}$

Hemivertebrae are less common in Pug dogs than other brachycephalic breeds ${ }^{11}$ and their association with neurological deficits remains unclear. ${ }^{10}$ Although some reports suggested the association between a kyphotic Cobb angle higher than $35^{\circ}$ and neurological deficits in brachycephalic screwtailed dog breeds with at least one vertebral malformation, ${ }^{11,15}$ this was not found in Pug dogs in another study. ${ }^{10}$ The presence of spondylosis deformans in combination with hemivertebrae has been found to be associated with neurological deficits in Pug dogs; chronic vertebral instability has been implicated in the pathogenesis, although further dynamic spinal imaging studies are necessary to support this hypothesis. ${ }^{10}$

In a previous report regarding canine spinal arachnoid diverticulum, spinal pathologies such as hemivertebra, disc protrusions or spondylosis were identified in the immediate vicinity of the spinal arachnoid diverticulum. ${ }^{6}$ The presence of caudal articular process dysplasia was not investigated in this study. Surgical decompression was performed with short-term neurological deterioration in three of the four of the dogs postoperatively. It is not known whether vertebral stabilization would have improved the outcome in that study. As the potential recurrence of neurological deficits after surgery for spinal arachnoid diverticulum is expected to occur after a median time of 20.5 months, ${ }^{1}$ the short long-term follow-up and the lack of postoperative imaging would prevent further conclusions from the aforementioned surgical study. ${ }^{6}$

Thoracolumbar intervertebral disc protrusion has been reported to result in disc-associated dynamic compression. ${ }^{16,17}$ Stress myelography ${ }^{16}$ was considered an acceptable diagnostic tool, and hemilaminectomy with vertebral stabilization was an effective treatment resulting in longterm neurological improvement in all dogs. Vertebral stabilization has been hypothesized to prevent further disc protrusion, promote disc atrophy and prevent any dynamic component of spinal cord compression. ${ }^{18}$

A poor long-term outcome with surgical management of spinal arachnoid diverticulum was described in Pug dogs, 1,19 although the cause of the poor long-term outcome was not investigated. A dynamic spinal cord compression was reported in dogs with thoracolumbar spinal arachnoid diverticulum, using stress myelography. ${ }^{20}$ Although myelographic stress study remains a somewhat subjective method for the evaluation of dynamic spinal cord compression, ${ }^{16,20}$, long-term neurological improvement after vertebral stabilization was noted in three of five dogs. ${ }^{20}$ The presence of an intramedullary lesion on magnetic resonance imaging (MRI) adjacent to spinal arachnoid diverticulum was suspected to lead to a worse outcome in the remaining two dogs. ${ }^{20}$

A poor surgical outcome was also reported for constrictive myelopathy in Pug dogs with caudal articular process dysplasia; however, this study did not record the number of dogs that underwent vertebral stabilization. ${ }^{8}$ Surgery to address pia-arachnoid fibrosis via subarachnoid-subarachnoid shunt placement has been previously described ${ }^{9}$; however, clinical signs continued to progress. Although this study considered caudal articular process dysplasia and vertebral instability as predisposing factors for pia-arachnoid fibrosis formation, they were only investigated and ruled out via radiography.

We hypothesized that Pug dogs diagnosed with T3 to L3 myelopathy and with intramedullary hyperintensity at the same level of thoracolumbar caudal articular process dysplasia had neurological signs in association with dynamic spinal cord compression or vertebral instability. Therefore, vertebral stabilization in isolation or with the treatment of the concurrent compressive pathologies (such as spinal arachnoid diverticulum and intervertebral disc protrusion) would reduce the recurrence of the disease and ameliorate clinical signs.

\section{Materials and Methods}

\section{Imaging Technique}

Patients underwent routine MRI scanning of the thoracolumbar spine using a high-field scanner (1.5T; Siemens Symphony Tim system, Erlangen Germany) under general anaesthesia in dorsal recumbency. The imaging protocol included sagittal and transverse plane on T2-weighted images (repetition time [TR] $3650 \mathrm{~ms}$, echo time [TE] $100 \mathrm{~ms}$, field of view [FOV] $280 \mathrm{~mm}$, Matrix $512 \times 448$ ), sagittal and transverse planes on T1weighted images (TR 550ms, TE $12 \mathrm{~ms}$, FOV $280 \mathrm{~mm}$, matrix $512 \times 448)$; dorsal plane in short-tau inversion recovery images (TR $3400 \mathrm{~ms}$, TE 40ms, FOV $300 \mathrm{~mm}$, matrix $384 \times 270$ ). To provide a better contrast between cerebrospinal fluid and other surrounding structures such as arachnoid webs and arachnoid adhesions, the three-dimensional constructive interference in steady state (3D-CISS) images were acquired in a transverse plane(TR $11.5 \mathrm{~ms}$, TE $5.75 \mathrm{~ms}$, FOV 170 , Matrix $256 \times 256$, slice thickness $1 \mathrm{~mm}$ ). With the aid of the multiplanar reconstruction software integrated to the MRI scanner, 3D-CISS data were further processed in sagittal and dorsal planes. Computed tomography (CT) examinations were performed of the entire thoracolumbar spine using a 160-slice scanner (Aquilion Prime Toshiba, Otawara Japan) with dogs positioned in dorsal recumbency, using the following exposure parameters: $120 \mathrm{kV}, 120$ to $150 \mathrm{mAs}, 200 \mathrm{~mm}$ FOV. The raw data were processed using the bone algorithm, which was 
subsequently evaluated in three orthogonal planes and threedimensional volumetric reconstruction.

The MRI spinal cord abnormalities were recorded according to widely accepted descriptions. Thus, an increase in intra-medullary T2-weighted signal intensity at the affected level represented oedema, pre-syrinx and/or gliosis ${ }^{21}$; an intra-dural extra-medullary lesion represented spinal arachnoid diverticulum, if a significant expansion of the subarachnoid space containing a fluid with signal identical to cerebrospinal fluid distorted the normal shape of the spinal cord and subarachnoid space, causing spinal cord compres$\operatorname{sion}^{22}$; an intra-dural extra-medullary lesion represented pia-arachnoid fibrosis, if multiple linear hypo-intense bands appeared to transverse the arachnoid space ${ }^{9}$; an extra-dural compression represented intervertebral disc protrusion, if bulging of the annulus fibrosus and dorsal longitudinal ligament was present. Intervertebral disc protrusion was considered severe, when the subarachnoid space and epidural fat were focally absent and the outline of the spinal cord was distorted and mild, when subarachnoid space and epidural fat were absent without spinal cord distortion. ${ }^{23}$

\section{Case Selection}

Pug dogs were retrospectively recruited from electronic patient records at Fitzpatrick referrals between 2013 and 2018. Inclusion criteria were Pug dogs that were presented with clinical signs compatible with a T3 to L3 myelopathy; MRI confirming the cause of thoracolumbar myelopathy and CT confirming caudal articular process dysplasia occurred at the same level as the MRI abnormalities, and record of surgical management that included vertebral stabilization in isolation or with concurrent spinal cord decompression.

Patient data collected included patient age, sex, neutering status, other spinal surgery performed prior to referral, duration of clinical signs (months), the presence or absence of urinary or faecal incontinence and the neurological status before and after surgical intervention. This was classified based on the Texas Spinal Cord Injury Scale (TSCIS). ${ }^{24}$

\section{Surgical Technique}

The surgical procedure was chosen by the primary surgeon according to the lesion localization and imaging appearance. Therefore, we divided the cases in three surgical groups: intervertebral disc protrusion, spinal arachnoid diverticulum and pia-arachnoid fibrosis.

The intervertebral disc protrusion group included only cases with severe extradural compression due to intervertebral disc protrusion associated with intramedullary hyperintensity at the same level of caudal articular process dysplasia. These were managed with decompression (hemilaminectomy or mini-hemilaminectomy with partial lateral corpectomy) and vertebral stabilization. The spinal arachnoid diverticulum group included cases with intradural compression due to spinal arachnoid diverticulum associated with intramedullary hyperintensity at the same level of caudal articular process dysplasia. These were managed with dorsal laminectomy, dorsal mid-line durotomy, dural marsupialization and vertebral stabilization. The pia-ara- chnoid fibrosis group included cases affected by intramedullary hyperintensity only or associated with subarachnoid fibrous bands at the same level of caudal articular process dysplasia. These cases were managed with vertebral stabilization only due to the absence of spinal cord compression.

Vertebral stabilization was performed using Imexx miniature threaded interface pins, enshrouded in a bolus of tobramycin impregnated polymethylmethacrylate (PMMA) bone cement. All cases underwent postoperative CT at the time of the surgery and occasionally at 6 weeks or 6 months postoperative. All cases had perioperative treatment with corticosteroids at an anti-inflammatory dose $(\sim 0.5 \mathrm{mg} / \mathrm{kg}$ once daily) and underwent a rehabilitation programme under the supervision of a chartered physiotherapist.

Postoperative follow-up was obtained through re-examination by the surgeon. Clinical records from re-examinations were available at the immediate postoperative period, at the discharge from the hospital and at a variable time during the postoperative period ( - Table 1 ). Follow-up phone interviews were obtained by one of the authors (AT). Questions were directed to gain information on the ambulatory status, presence of discomfort and level of exercise compared with the preoperative status. Additional details on urinary or faecal incontinence and cause of death (when applicable) were also recorded.

\section{Outcome}

Outcome was classified as excellent, if no signs of neurological dysfunction were found on follow-up examinations and the owner perceived the dog was clinically normal, with no restriction in physical activities. Outcome was considered good, if minimal signs of neurological dysfunction were found on follow-up examinations and the owner perceived the dog had improved but showing some restrictions in physical activities. Outcome was considered satisfactory, if moderate signs of neurological dysfunction were found on follow-up examinations and the owner perceived the dog was the same as prior surgery. Outcome was poor, if the dog failed to improve or continued to deteriorate. Outcome was also considered poor for cases where the euthanasia was elected due to comorbidities.

\section{Results}

Fourteen cases were eligible for inclusion in this study (-Tables 2 and 3). Median age of presentation was 8.5 years (range: $2-11.8)$. Sex of the dogs were entire male $(n=5)$, neutered male $(n=4)$, entire female $(n=1)$ and neutered female $(n=4)$. All Pug dogs displayed varying degree of spastic paraparesis and pelvic limb ataxia at the time of initial presentation with a median TSCIS of 7 out of 10 (-Table 1). Seven cases had urinary or faecal incontinence, or both, prior to vertebral stabilization (-Table 2). The median duration of clinical signs prior to presentation to our referral hospital was 6.5 months (range: $0.4-30$ ). The median follow-up obtained from re-examination was 4.9 months (range: $2.1-9.8$ ) and via phone interview was 13.3 months (range: $10-24$ ). 


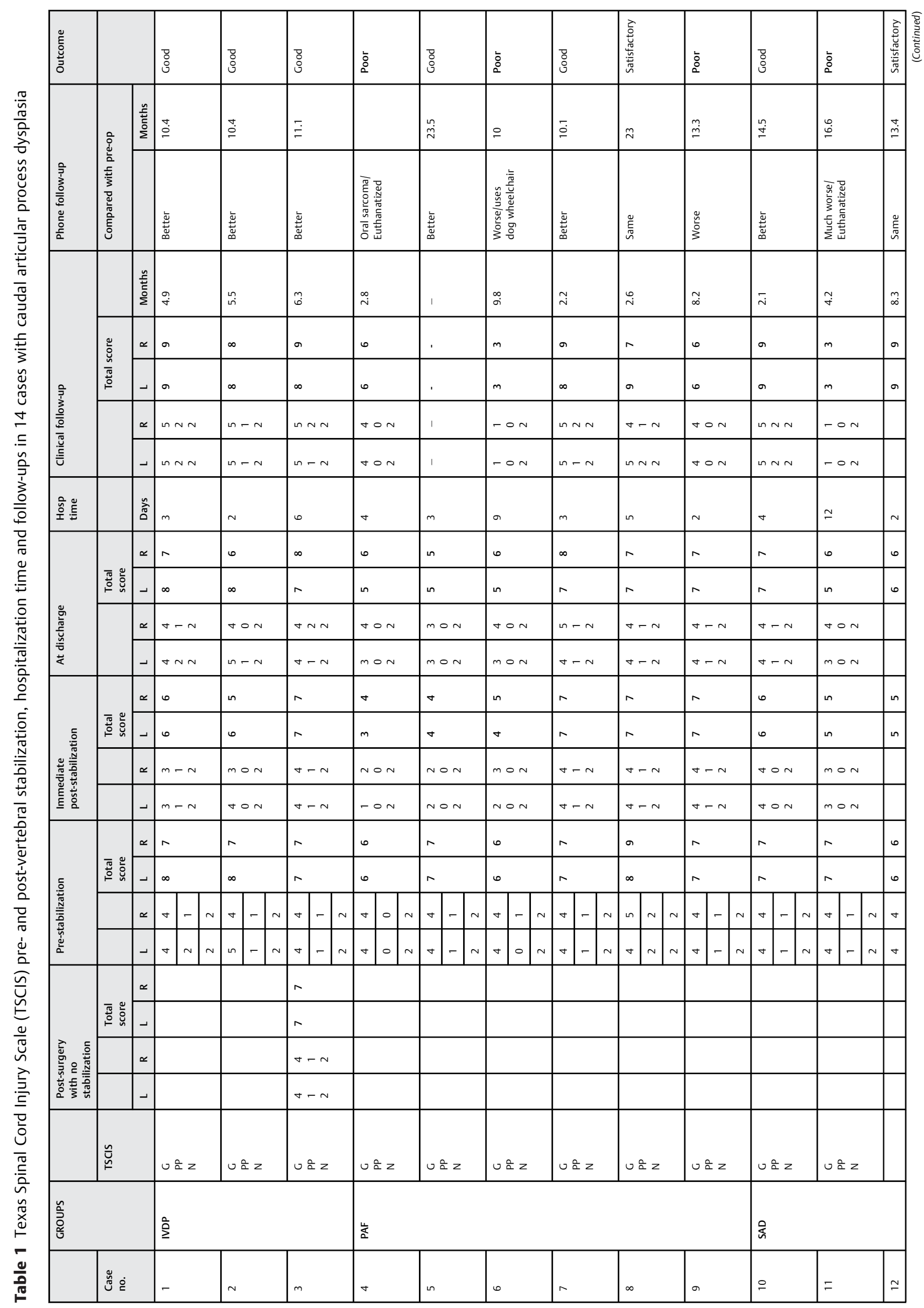




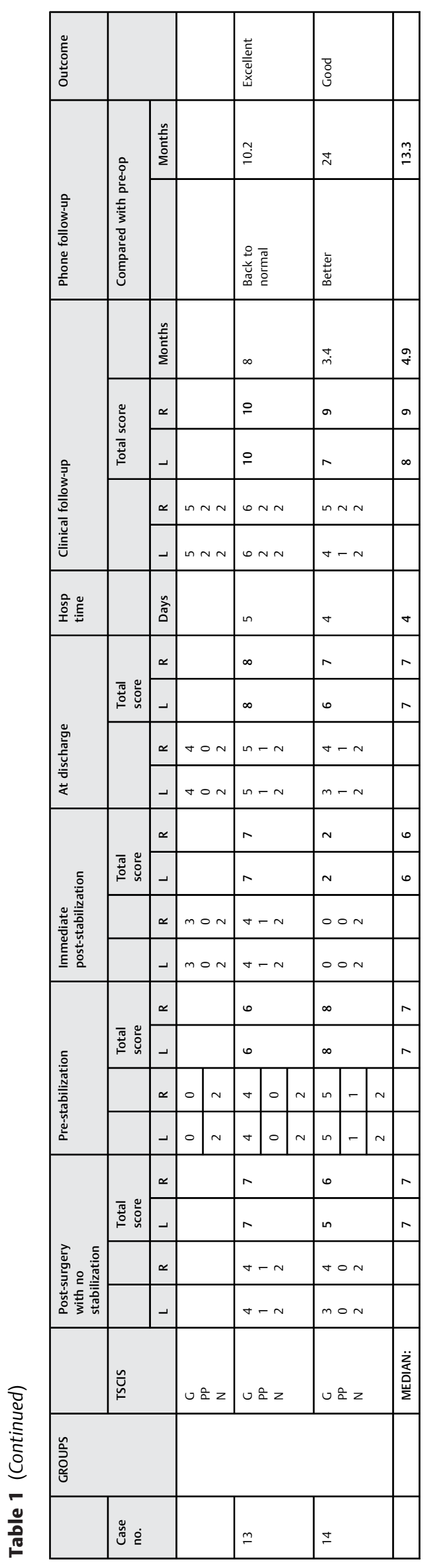

\section{MRI Findings}

Caudal articular process dysplasia was localized between $\mathrm{T} 10$ and L1 ( - Table 2). In all cases, there was an intramedullary lesion at the level of caudal articular process dysplasia evident as hyperintense signal on $\mathrm{T} 2$-weighted image (T2WI). Five cases showed intervertebral disc protrusion, but only in three cases it was associated with extra-dural spinal cord compression ( - Fig. $\mathbf{1}$ ). Nine cases had a relevant intra-dural extra-medullary lesion identified on T2WI and 3D-CISS sequences, which was suspected to represent piaarachnoid fibrosis in four cases and spinal arachnoid diverticulum in five cases. Two cases showed mainly intramedullary signal changes without evident spinal cord compression (-Table 2). Nine cases had other congenital vertebral malformations such as transitional vertebrae and abnormal number of vertebrae and two cases showed kyphoscoliosis following the first surgical procedure performed without vertebral stabilization ( - Table 3 ).

\section{Surgical Treatment}

Surgical treatments are summarized in $\mathbf{- T a b l e s} \mathbf{2}$ and $\mathbf{3}$. Of the fourteen Pug dogs that underwent vertebral stabilization, three underwent decompressive procedures prior to vertebral stabilization via partial lateral corpectomy (intervertebral disc protrusion group), six underwent vertebral stabilization only (pia-arachnoid fibrosis group) and five underwent dorsal laminectomy for spinal arachnoid diverticulum marsupialization prior to vertebral stabilization (spinal arachnoid diverticulum group). Five cases had bilateral stabilization, while the other nine cases had a unilateral stabilization (- Table $\mathbf{3}$ ). The selection of unilateral or bilateral fixation was based on surgeon preference; the main indication for unilateral stabilization was to limit surgical dead space over a dorsal laminectomy defect, due to the presence of a PMMA bolus. All dogs had postoperative CT, two dogs had imaging at 6 weeks (cases 3 and 6 ) and two dogs at 6 months (cases 9 and 12).

Three cases (case 3,13 and 14) (-Tables 2 and 3) had undergone previous surgical interventions without the identification of caudal articular process dysplasia: Cases 3 (-Fig. 2) and 13 (-Fig. 3) had previously undergone decompression (durotomy and marsupialization or durectomy) for spinal arachnoid diverticulum, with signs related to thoracolumbar myelopathy present for over 5.5 months. Due to the further neurological deterioration, spinal MRI and CT scan were performed 18 and 7 months (in cases 3 and 13 respectively) after the first surgical intervention. These confirmed the presence of caudal articular process dysplasia in both cases. Case 3 developed intervertebral disc protrusion and mild kyphoscoliosis at the previous surgical site; while in case 13 , recurrence was attributed to reformation of the spinal arachnoid diverticulum. Partial lateral corpectomy and vertebral stabilization were performed in case 3; while dorsal spinal arachnoid diverticulum marsupialization and spinal stabilization were performed in case 13. Case 14 (-Fig. 4) underwent hemilaminectomy to treat a suspected intervertebral disc protrusion with signs related to thoracolumbar myelopathy present for over a month. Following a 


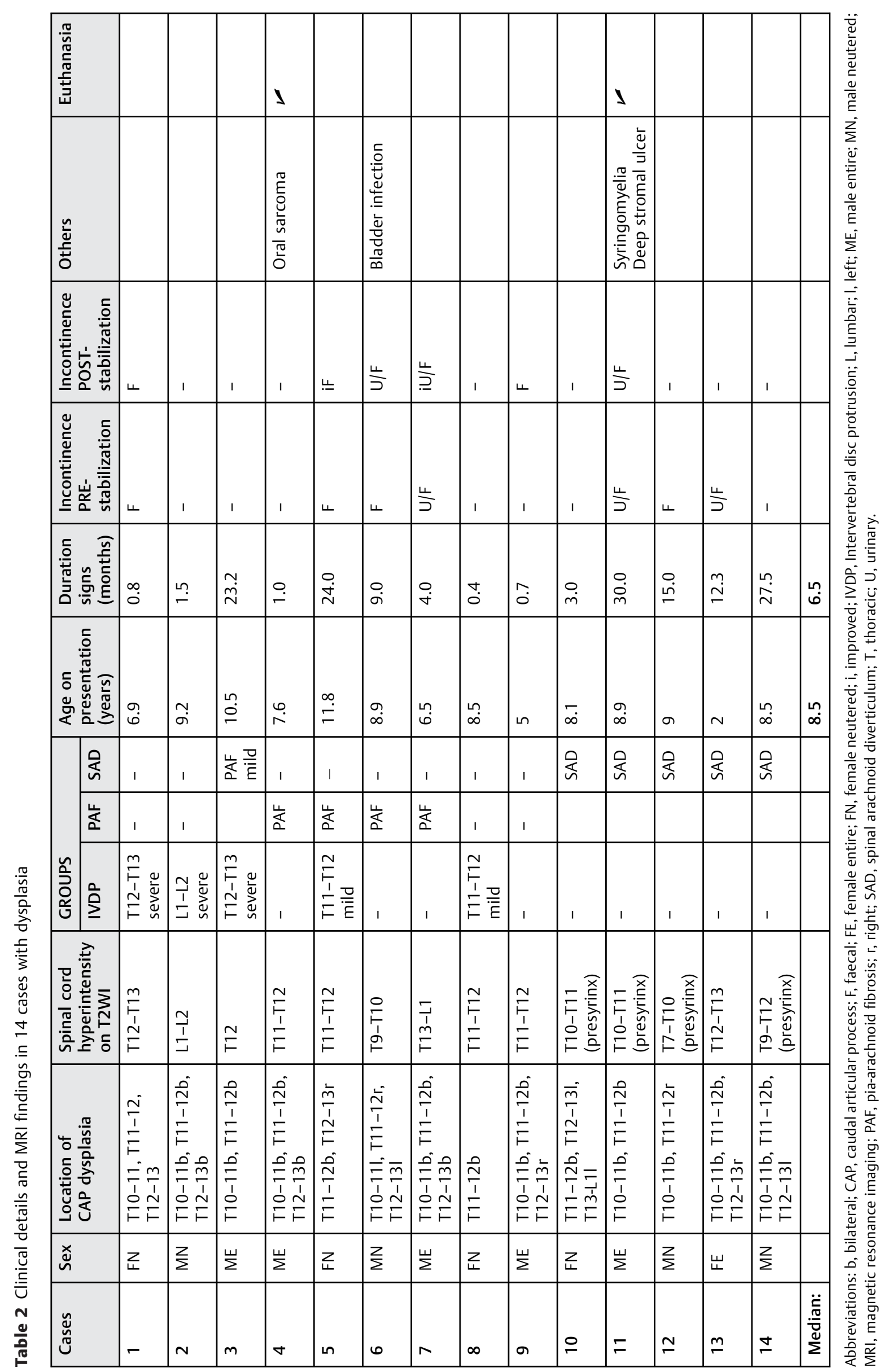




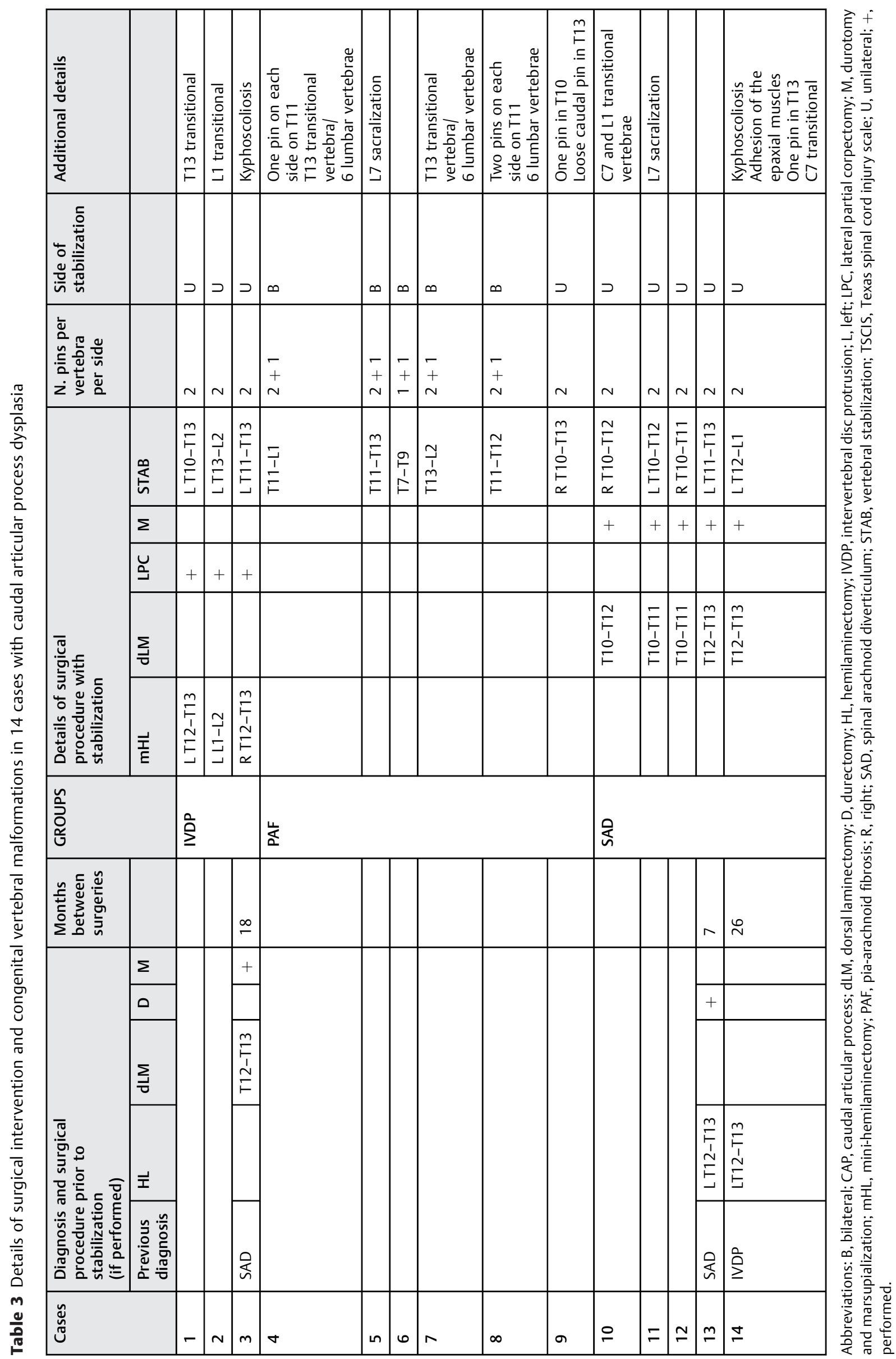



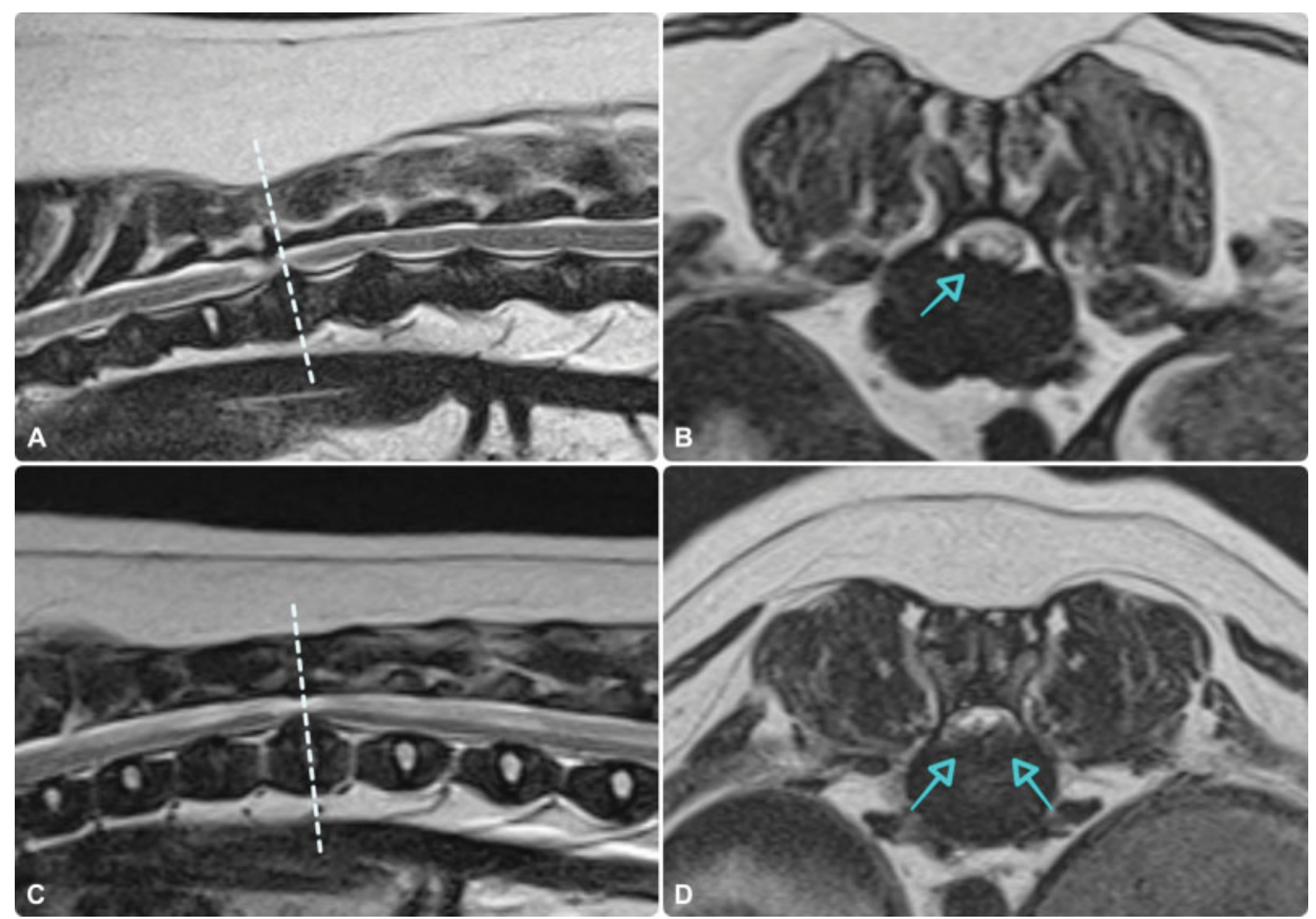

Fig. 1 Case 5: mid-sagittal and transverse T2-weighted (T2W) (A and B) images at the level of T11 to T12 intervertebral disc space showing a mild intervertebral disc protrusion (IVDP) (blue arrow) at the level of caudal articular process dysplasia. Case 1: mid-sagittal and transverse T2W (C and D) images at the level of T12 to T13 intervertebral disc space showing severe IVDP (blue arrows) at the level of caudal articular process dysplasia. (Siemens Symphony Tim system, 1.5 T, Erlangen, Germany).

reported short-lived (2-4 weeks) amelioration of the clinical signs, the dog demonstrated a progressive deterioration of clinical signs. Spinal CT and MRI scans performed 26 months after the first surgical intervention supported the presence of caudal articular process dysplasia and spinal arachnoid diverticulum adjacent to the region of the previous hemilaminectomy. Mild kyphoscoliosis and the adhesion of the epaxial muscles to dura mater at the previous surgical site were present. Spinal arachnoid diverticulum marsupialization and vertebral stabilization were performed in this case (-Table $\mathbf{2}$ and $\mathbf{3}$ ).

\section{Surgical Outcome}

In all cases that underwent vertebral stabilization, we observed transient neurological deterioration (TSCIS: median of 6 out of 10) in the immediate postoperative period with a tendency to return to the preoperative status by discharge (TSCIS: median of 7 out of 10 ) (-Table $\mathbf{1}$ ). In 10 cases, we detected neurological improvement (TSCIS: median 8.5 out of 10) at the clinical follow-up at 4.9 months and phone interview follow-up at 13.3 months. Median hospitalization time was 4 days (range: $2-12$ ).

Overall, one case had an excellent outcome, seven cases had a good surgical outcome and two cases had a satisfactory outcome. Four cases had a poor outcome. Case 4 (-Table 2) was euthanatized for an unrelated cause (a poorly differentiated soft tissue sarcoma in the oral cavity) $\sim 7$ months postsurgical intervention. During the postoperative period, the dog remained neurologically stable ( $\mathbf{- T a b l e ~} \mathbf{1}$ ). Case 11 was euthanatized 4 months post-surgery due to the lack of neurological improvement. This case had a long duration of clinical signs before presentation (30 months) (-Table 2). This dog was diagnosed with concurrent syringomyelia at the level of T1 to T5 vertebrae with a maximum transverse syrinx width of $6 \mathrm{~mm}$, became paraplegic immediately postoperatively ( - Table 1) and developed a deep stromal corneal ulcer during the postoperative period, which delayed the onset of rehabilitation. The owner elected for euthanasia. Case 9 had loosening of one of the caudal pins at a 6 -month postoperative CT (-Table 3) and a slight deterioration of the neurological status was reported. This dog had only one pin was inserted in T10 ( - Table 3) due to a technical challenge: the length of the vertebral fusion, that is, over four vertebral bodies in combination with the placement of only one pin in the cranial portion may have contributed to the failure of the implant. Surgical revision was declined. Case 6 demonstrated neurological deterioration at the clinical and phone interview follow-ups, becoming non-ambulatory paraparetic (-Table 1 ). However, 

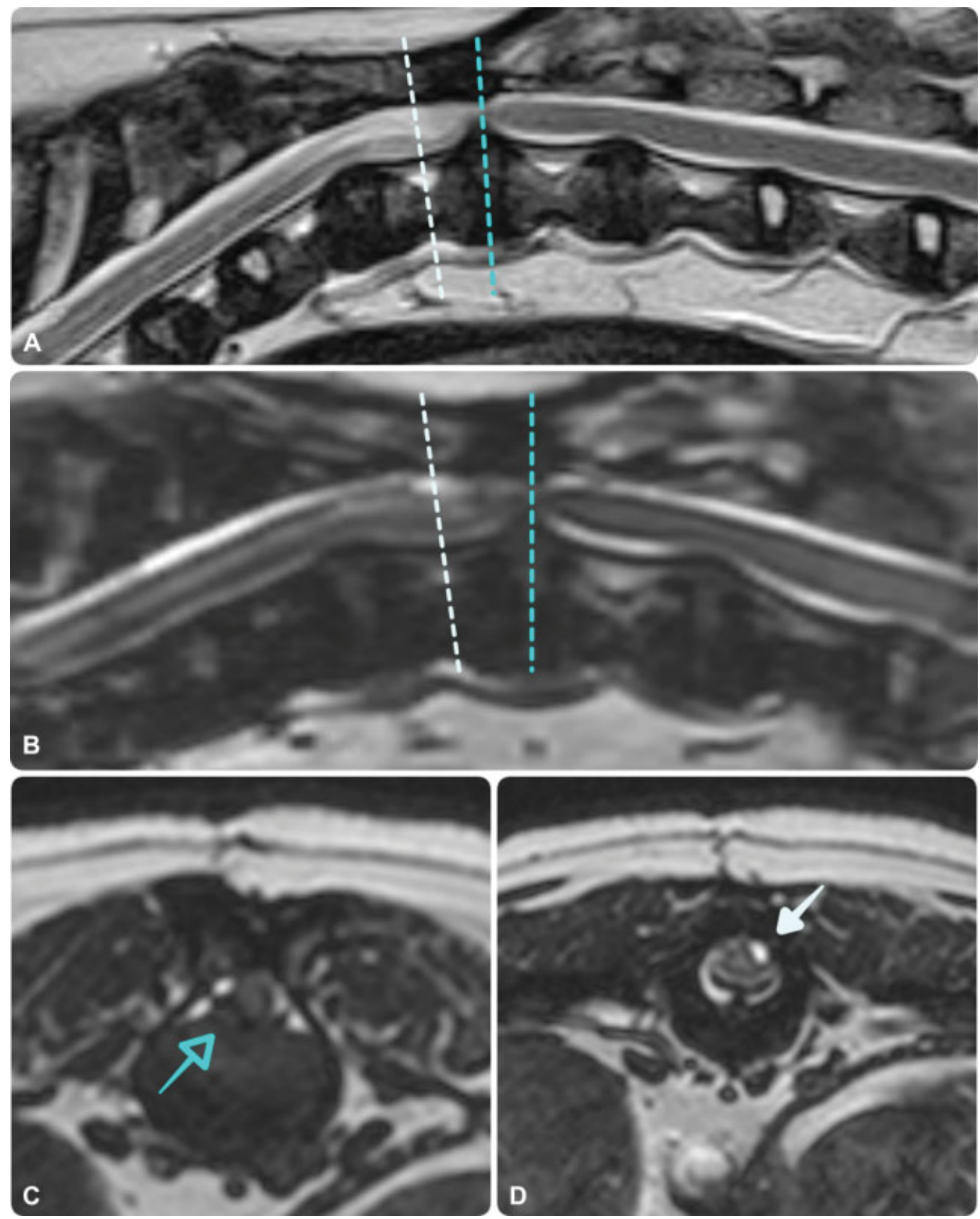

Fig. 2 Case 3: mid-sagittal T2-weighted (T2W) and three-dimensional constructive interference in steady state (3D-CISS) (A and B) and transverse T2W and 3D-CISS (C and D) images at the level of T12 to T13 intervertebral disc space demonstrating the recurrence of spinal arachnoid diverticulum (SAD) (white arrow), intervertebral disc protrusion (blue arrow) and kyphoscoliosis following SAD surgery. (Siemens Symphony Tim system, 1.5 T, Erlangen, Germany).

this dog showed only a slight deterioration at discharge and spinal CT performed approximately 6-week after surgery demonstrated adequate implant positioning (-Table 1). A severe urinary tract infection developed within the postoperative period and urinary incontinence were reported to have contributed to the poor outcome.

Following surgical intervention, urinary and faecal incontinence completely resolved in only two cases (cases 12 and 13), while in two cases (case 5 and 7) its frequency was reported to have slightly reduced. Faecal incontinence developed in one case (case 9). Urinary or faecal incontinence persisted in the remaining three cases (cases $1,11,6$ ) (-Table $\mathbf{2}$ ).

\section{Discussion}

Our study was to investigate whether vertebral stabilization was associated with the resolution or amelioration of clinical signs in Pug dogs diagnosed with thoracolumbar myelopathy, and with intramedullary hyperintensity present at the level of thoracolumbar caudal articular process dysplasia.

Overall, we observed neurological improvement in 10 cases. The chronicity of the clinical signs may have contributed to the persistent of neurological deficits.

Case 13 was the only case with excellent outcome. This was also the youngest Pug dog (-Table 2) and therefore 

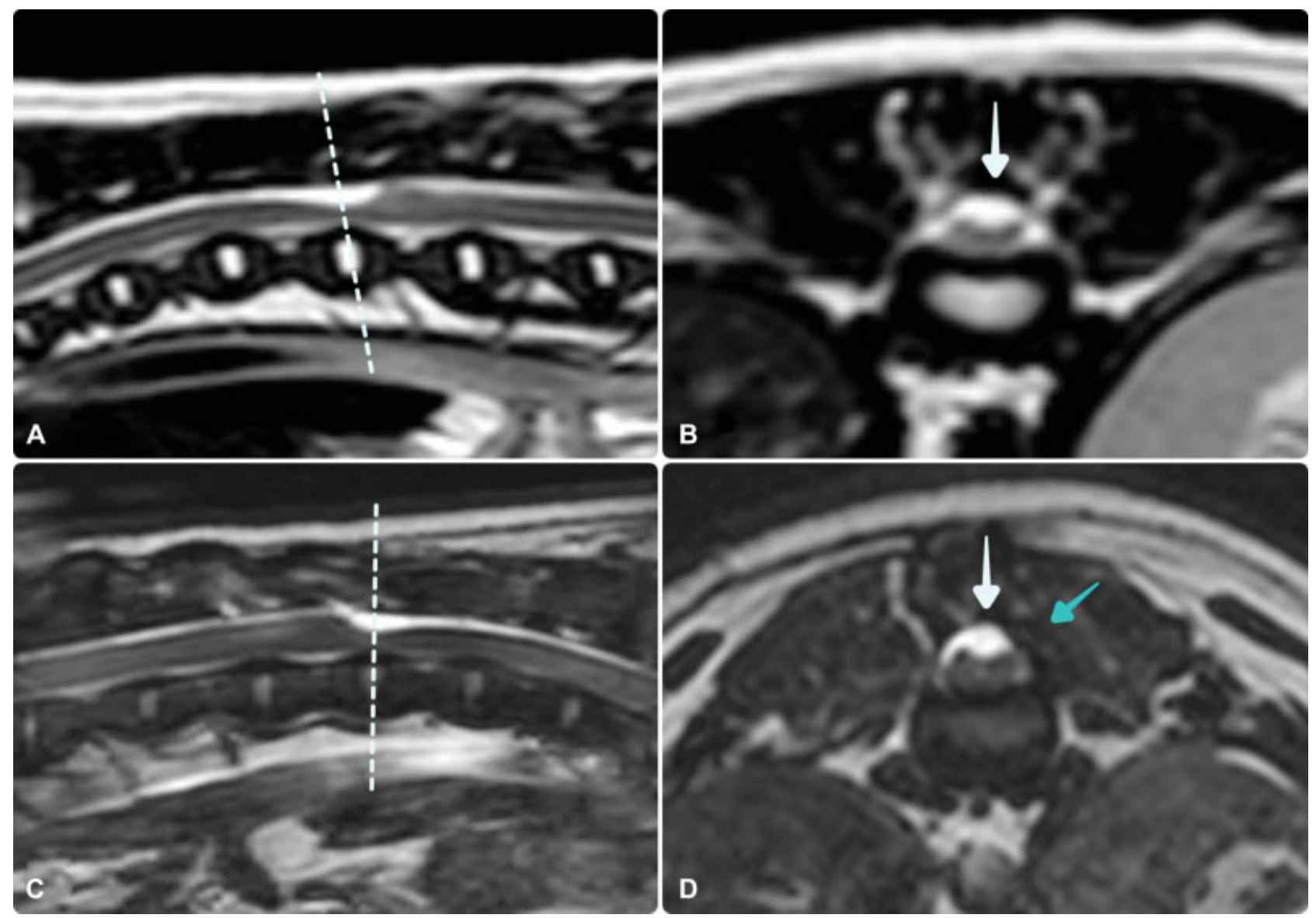

Fig. 3 Case 13: mid-sagittal and transverse T2-weighted (T2W) (A and B) performed at another referral hospital prior to hemilaminectomy to treat spinal arachnoid diverticulum (SAD) (white arrow) and mid-sagittal and transverse three-dimensional constructive interference in steady state ( $C$ and $D$ ) images post-hemilaminectomy without vertebral stabilization at the level of T12 to T13 intervertebral disc space demonstrating the laminectomy site (blue arrow) and the recurrence of SAD (white arrow) (Siemens Symphony Tim system, 1.5 T, Erlangen, Germany).

spinal arachnoid diverticulum was considered to be developmental rather than acquired. Caudal articular process dysplasia was initially undiagnosed and we speculated that the neurological progression was potentially associated with vertebral instability following hemilaminectomy ( - Table 3 ). The dog showed clinical improvement, when vertebral stabilization was added to the surgical planning.

Among the group of dogs with a good outcome, two cases (case 3 and 14) with undiagnosed caudal articular process dysplasia had surgical intervention to address spinal arachnoid diverticulum and intervertebral disc protrusion respectively. In these cases, the signs related to the thoracolumbar myelopathy recurred and kyphoscoliosis developed at the surgical site in association with intervertebral disc protrusion. Kyphoscoliosis may be secondary to the progression of the disease or increased vertebral instability following the surgical procedure. The vertebral stabilization subsequently performed appeared to ameliorate neurological progression.

The significance of caudal articular process dysplasia in our study population remains controversial and why some dogs with caudal articular process dysplasia should develop spinal cord disease at the affected level requires further investigation. We speculated that surgical interventions such as facetectomy, hemi or dorsal laminectomy to approach the spinal cord at the same site of caudal articular process dysplasia might exacerbate vertebral instability (-Fig. 5), leading to kyphoscoliosis and progression of the disease. Hence, the presence of caudal articular process dysplasia should be considered in surgical planning and vertebral stabilization may be indicated.

Previous studies had shown an association between abnormal gait and age ${ }^{25}$ and the presence and severity of caudal articular process dysplasia with the occurrence of intervertebral disc protrusion in aged ( $>8$ years old) Pug dogs (Nishida $\mathrm{H}$, Nakata S, Maeda S, Kamishina H. Characteristics of the caudal articular process abnormalities in Pugs with thoracolumbar disk herniation. Poster 44 in ESCN/ECVN congress proceedings, Helsinki, Finland, 2017). In our study, we found that the median age of onset of clinical signs was 8.5 years; therefore, Pug dogs may not show signs related to caudal articular process dysplasia until they are older. ${ }^{10}$ Previous reports hypothesized loss of stability of the vertebral column secondary to caudal articular process dysplasia ${ }^{13}$ and found the presence of intramedullary lesion on MRI associated with a worse outcome. ${ }^{20}$ We suspected that intramedullary lesion in our study may be secondary to chronic vertebral instability which overtime may lead to the developing of other concurrent disorders such as piaarachnoid fibrosis, spinal arachnoid diverticulum and 

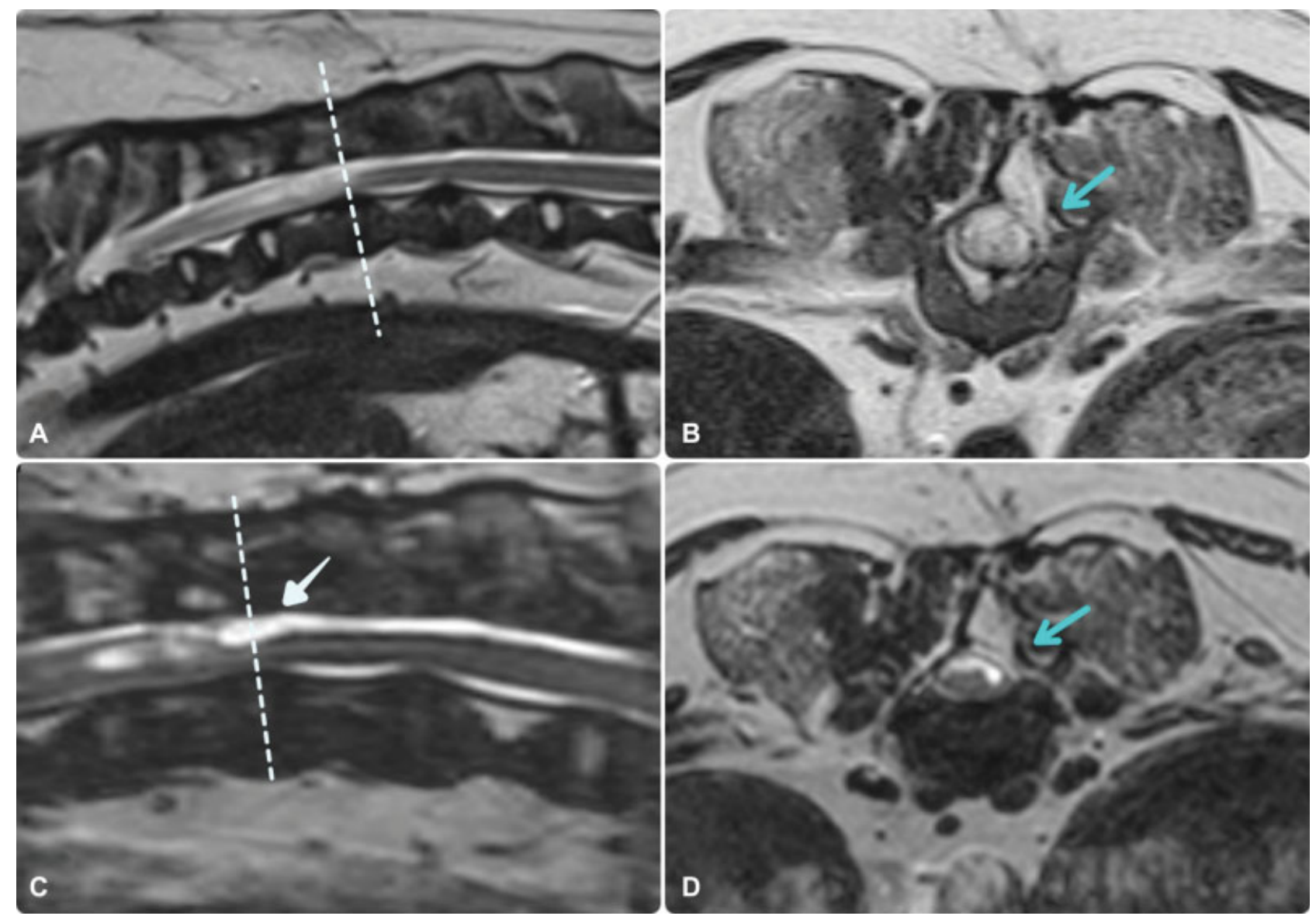

Fig. 4 Case 14: mid-sagittal and transverse T2-weighted (T2W) (A and B) and mid-sagittal and transverse three-dimensional constructive interference in steady state ( $C$ and $D$ ) images at the level of T12 to T13 intervertebral space demonstrating the recurrence of SAD (white arrow), mild kyphoscoliosis and the adhesion of the epaxial muscles to dura mater at the previous surgical site (blue arrow) (Siemens Symphony Tim system, 1.5 T, Erlangen, Germany).

intervertebral disc protrusion at the same level. We would therefore speculate that to reduce the prognostic influence of the chronicity of the clinical signs, early vertebral stabilization could be indicated when intramedullary hyperintensity is diagnosed at the level of caudal articular process dysplasia. However, further studies are necessary to support this speculation.

We identified nine Pug dogs with other congenital vertebral malformations such as transitional vertebrae and abnormal number of vertebrae ( $\mathbf{- T a b l e ~} \mathbf{3}$ ). In accordance to previous studies, the common presence of congenital vertebral malformations may be considered an undirect cause of the neurological dysfunction ${ }^{10}$ as the alteration in vertebral biomechanics may contribute to the aetiopathogenesis. ${ }^{11,26}$

Although MRI may occasionally demonstrate caudal articular process abnormalities, the spatial resolution of these small structures is often poor and CT should be used as a preferred imaging modality to identify the presence of this malformation ${ }^{10}$ (-Fig. 6). Our findings support the usefulness of both MRI and CT in the preoperative assessment of Pug dogs with thoracolumbar myelopathy.

Surgical outcome was considered poor in four cases (29\%) ( - Table 1): Case 4 remained stable during the postoperative period; however, the follow-up was relatively short due to the concurrent oral sarcoma. Case 11 showed chronic clinical signs before presentation and had a delay in commencing the rehabilitation programme due to other comorbidities. Surgical manipulation of a chronically injured spinal cord and/or recurrence of spinal arachnoid diverticulum may also have contributed to the reported deterioration. Unfortunately, CT was not repeated in this case. The presence of syringomyelia concurrent with spinal arachnoid diverticulum has been previously associated with a negative prognosis ${ }^{20,27,28}$ and has likely further compromised the recovery in this case. The decision of performing unilateral or bilateral vertebral stabilization did not adversely influence the outcome or cause any specific types of complications. However, the authors preferred the unilateral approach for stabilization in the presence of a dorsal laminectomy defect to decrease dead space and the risk of postoperative compressive haematoma/ seroma formation. Case 6 demonstrated further progression of neurological dysfunction; however, CT myelogram was not performed in this case and the possible recurrence of spinal arachnoid diverticulum formation, chronicity of the condition or reperfusion injury remains a speculation.

Incontinence may persist despite the amelioration of the neurological dysfunction (-Table 1). Although it is unclear the reason why the incontinence developed in some dogs 


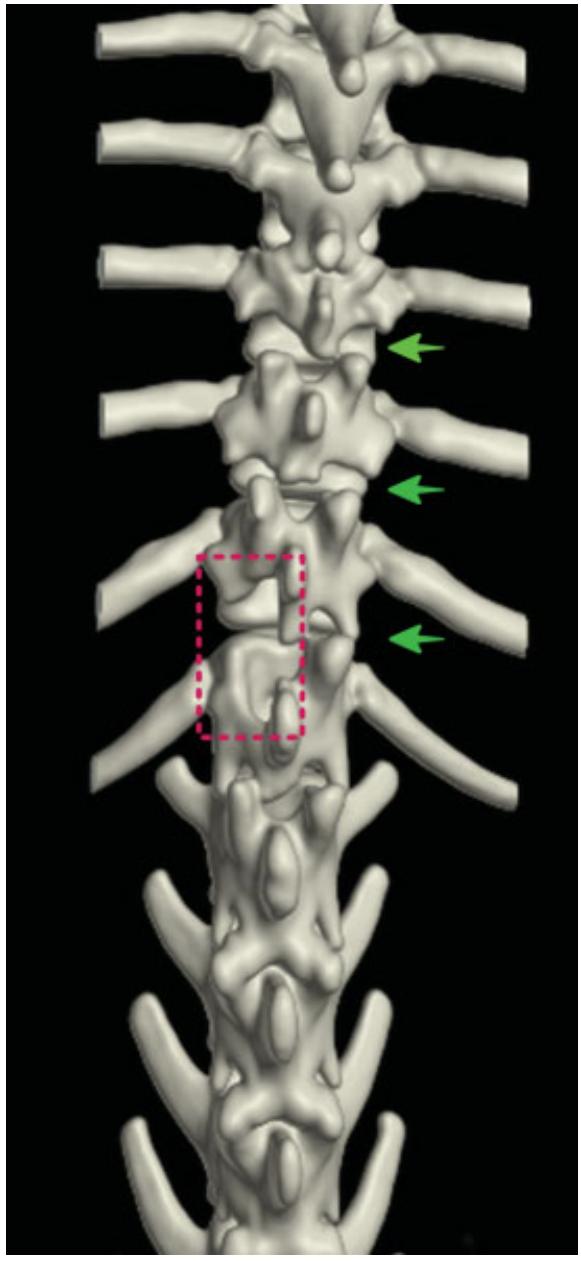

Fig. 5 Case 13: dorsal view of the three-dimensional reconstruction computed tomography showing T12 to T13 previous hemilaminectomy surgery (red square) performed prior the diagnosis of caudal articular process dysplasia (green arrows). (Toshiba Aquilion PRIME 160-slice multislice CT scanner, Japan). rather than others, this could influence the overall outcome. Hence, we would recommend performing vertebral stabilization sooner in the course of the disease, to prevent or minimize permanent neurological deficits.

Limitations of this study include its retrospective nature, the small sample size, the lack of control cases and dynamic studies and the length and nature of follow-up data gathering. Owner financial constraint was also a limiting factor in the availability of CT imaging in the postoperative period. Postoperative MRI would be ideal in evaluating spinal cord and arachnoid space in dogs that show neurological deterioration; however, the use of stainless-steel implants prevents this because of imaging artefacts arising from their ferromagnetic properties. Although myelo-CT can assist in evaluating the arachnoid space following surgical intervention; the increased use of titanium alloy screws and pins and the development of metal artefact reduction MRI sequences may help in the future to reduce the size and intensity of susceptibility artefacts, resulting from magnetic field distortion, and increase anatomical visibility and diagnostic confidence level. ${ }^{29-31}$

We considered vertebral stabilization in isolation or with concurrent spinal cord decompression to treat a plethora of conditions which aetiopathogenesis is not well understood. Future studies may further clarify the complexity of these conditions and propose novel treatments.

Finally, we would advise to perform both spinal MRI and $\mathrm{CT}$ in aged Pug dogs presented with progressive thoracolumbar myelopathy, and no obvious signs of discomfort. Both imaging modalities might enable the surgeon to choose the most appropriate surgical intervention. The findings of our study support the contention that vertebral stabilization in Pug dogs affected by caudal articular process dysplasia may help to ameliorate or at least halt the clinical signs associated with thoracolumbar myelopathy, which have previously been associated with a poor prognosis in this breed.
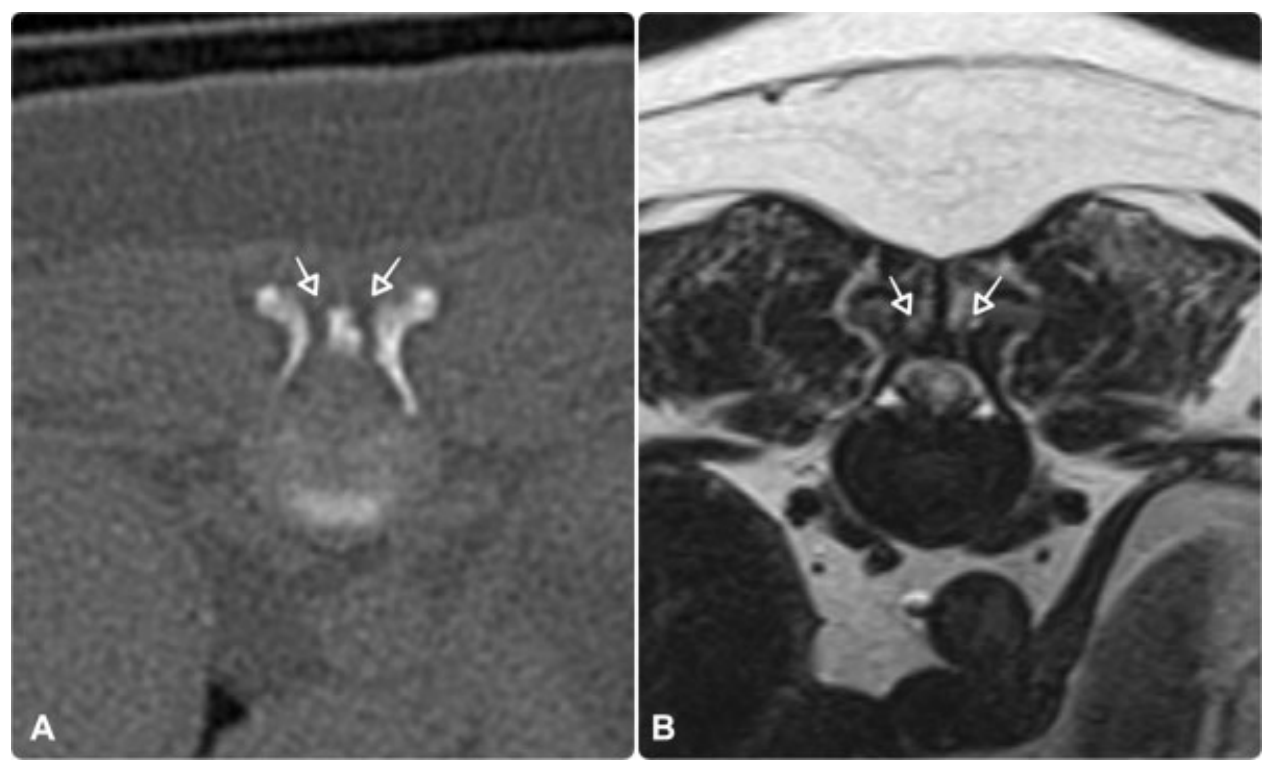

Fig. 6 Case 8: transverse computed tomography (CT) (A) and transverse T2-weighted image (B) at the level of T11 to T12 intervertebral disc space showing the challenge in diagnosing caudal articular process dysplasia (white arrows) on magnetic resonance imaging when compared with CT. (Siemens Symphony Tim system, 1.5 T, Erlangen, Germany; Toshiba Aquilion PRIME 160-slice multislice CT scanner, Japan). 


\section{Conflict of Interest}

None of the authors of this article has a financial or personal relationship with other people or organizations that could inappropriately influence or bias the content of the paper.

\section{Author Contribution}

Anna Tauro contributed to conception of study, study design, acquisition of data and data analysis and interpretation. Jeremy Rose and Clare Rusbridge contributed to study design and data analysis and interpretation. Colin John Driver contributed to conception of study, study design, and data analysis and interpretation. All authors drafted, revised and approved the submitted manuscript.

\section{References}

1 Alcoverro E, McConnell JF, Sanchez-Masian D, De Risio L, De Decker $\mathrm{S}$, Gonçalves R. Late-onset recurrence of neurological deficits after surgery for spinal arachnoid diverticula. Vet Rec 2018;182(13):380

2 Mauler DA, De Decker S, De Risio L, et al. Signalment, clinical presentation, and diagnostic findings in 122 dogs with spinal arachnoid diverticula. J Vet Intern Med 2014;28(01):175-181

3 Flegel T, Müller MK, Truar K, Löffler C, Oechtering G. Thoracolumbar spinal arachnoid diverticula in 5 pug dogs. Can Vet J 2013; 54(10):969-973

4 Bismuth C, Ferrand FX, Millet M, et al. Original surgical treatment of thoracolumbar subarachnoid cysts in six chondrodystrophic dogs. Acta Vet Scand 2014;56:32

5 Jeffery ND, Levine JM, Olby NJ, Stein VM. Intervertebral disk degeneration in dogs: consequences, diagnosis, treatment, and future directions. J Vet Intern Med 2013;27(06):1318-1333

6 Flegel T, Boettcher IC, Ludewig E, Kiefer I, Oechtering G, Böttcher P. Partial lateral corpectomy of the thoracolumbar spine in 51 dogs: assessment of slot morphometry and spinal cord decompression. Vet Surg 2011;40(01):14-21

7 Charalambous M, Jeffery ND, Smith PM, et al. Surgical treatment of dorsal hemivertebrae associated with kyphosis by spinal segmental stabilisation, with or without decompression. Vet J 2014; 202(02):267-273

8 Fisher SC, Shores A, Simpson ST. Constrictive myelopathy secondary to hypoplasia or aplasia of the thoracolumbar caudal articular processes in Pugs: 11 cases (1993-2009). J Am Vet Med Assoc 2013;242(02):223-229

9 Meren IL, Chavera JA, Alcott CJ, Barker AK, Jeffery ND. Shunt tube placement for amelioration of cerebrospinal fluid flow obstruction caused by spinal cord subarachnoid fibrosis in dogs. Vet Surg 2017;46(02):289-296

10 Rohdin C, Häggström J, Ljungvall I, et al. Presence of thoracic and lumbar vertebral malformations in pugs with and without chronic neurological deficits. Vet J 2018;241:24-30

11 Ryan R, Gutierrez-Quintana R, Ter Haar G, De Decker S. Prevalence of thoracic vertebral malformations in French bulldogs, Pugs and English bulldogs with and without associated neurological deficits. Vet J 2017;221:25-29

12 Bertram S, Ter Haar G, De Decker S. Caudal articular process dysplasia of thoracic vertebrae in neurologically normal French bulldogs, English bulldogs, and Pugs: prevalence and characteristics. Vet Radiol Ultrasound 2018;59(04):396-404
13 Bouma JL. Congenital malformations of vertebral articular processes in dogs. Vet Clin North Am Small Anim Pract 2016;46(02):307-326

14 Penderis J, Schwarz T, McConnell JF, Garosi LS, Thomson CE, Dennis R. Dysplasia of the caudal vertebral articular facets in four dogs: results of radiographic, myelographic and magnetic resonance imaging investigations. Vet Rec 2005;156(19):601-605

15 Guevar J, Penderis J, Faller K, Yeamans C, Stalin C, GutierrezQuintana R. Computer-assisted radiographic calculation of spinal curvature in brachycephalic "screw-tailed" dog breeds with congenital thoracic vertebral malformations: reliability and clinical evaluation. PLoS One 2014;9(09):e106957

16 Aikawa T, Shibata M, Sadahiro S. Hemilaminectomy and vertebral stabilization for thoracolumbar intervertebral disc associated dynamic compression in 11 dogs. Vet Comp Orthop Traumatol 2013;26(06):498-504

17 Downes CJ, Gemmill TJ, Gibbons SE, McKee WM. Hemilaminectomy and vertebral stabilisation for the treatment of thoracolumbar disc protrusion in 28 dogs. J Small Anim Pract 2009;50(10): 525-535

18 McKee WM, Downes CJ. Vertebral stabilisation and selective decompression for the management of triple thoracolumbar disc protrusions. J Small Anim Pract 2008;49(10):536-539

19 Alisauskaite N, Cizinauskas S, Jeserevics J, et al. Short- and longterm outcome and magnetic resonance imaging findings after surgical treatment of thoracolumbar spinal arachnoid diverticula in 25 Pugs. J Vet Intern Med 2019

20 Aikawa T, Shimatsu T, Miyazaki Y. Hemilaminectomy, diverticular marsupialization, and vertebral stabilization for thoracolumbar spinal arachnoid diverticula in five dogs. J Am Anim Hosp Assoc 2019;55(02):110-116

21 Rusbridge C, MacSweeny JE, Davies JV, et al. Syringohydromyelia in Cavalier King Charles spaniels. J Am Anim Hosp Assoc 2000;36 (01):34-41

22 Skeen TM, Olby NJ, Muñana KR, Sharp NJ. Spinal arachnoid cysts in 17 dogs. J Am Anim Hosp Assoc 2003;39(03):271-282

23 Brisson BA. Intervertebral disc disease in dogs. Vet Clin North Am Small Anim Pract 2010;40(05):829-858

24 Levine GJ, Levine JM, Budke CM, et al. Description and repeatability of a newly developed spinal cord injury scale for dogs. Prev Vet Med 2009;89(1-2):121-127

25 Rohdin C, Jäderlund KH, Ljungvall I, Lindblad-Toh K, Häggström J. High prevalence of gait abnormalities in pugs. Vet Rec 2018;182 (06):167

26 Brocal J, De Decker S, José-López R, et al. C7 vertebra homeotic transformation in domestic dogs - are Pug dogs breaking mammalian evolutionary constraints? J Anat 2018;233(02):255-265

27 Jurina K, Grevel V. Spinal arachnoid pseudocysts in 10 rottweilers. J Small Anim Pract 2004;45(01):9-15

28 Gonçalves R, Hammond G, Penderis J. Imaging diagnosis: erroneous localization of spinal arachnoid cyst. Vet Radiol Ultrasound 2008;49(05):460-463

29 Radzi S, Cowin G, Robinson M, et al. Metal artifacts from titanium and steel screws in CT, 1.5T and 3T MR images of the tibial Pilon: a quantitative assessment in 3D. Quant Imaging Med Surg 2014;4 (03):163-172

30 Talbot BS, Weinberg EP. MR imaging with metal-suppression sequences for evaluation of total joint arthroplasty. Radiographics 2016;36(01):209-225

31 Han SB, Yoon YC, Kwon JW. Comparison Study between Conventional Sequence and Slice-Encoding Metal Artifact Correction (SEMAC) in the diagnosis of postoperative complications in patients receiving lumbar inter-body fusion and pedicle screw fixation surgery. PLoS One 2016;11(10):e0163745 